\title{
Effect of fish oil, garlic oil supplementation and nutrition counselling on daily food and nutrient intake of at risk coronary heart subjects
}

\author{
NAVJOT KaUR
}

\begin{abstract}
A group of 90 male subjects aged 30-50 years who were at risk of coronary heart disease were purposively selected. Nutritional status was assessed using dietary survey, 24 hour recall cum weighment method. Subjects were followed for one month and no treatment was given except the prescribed medicines and were treated as self control. Subjects were divided into three groups of 30 subjects each. Supplementation with fish oil capsules $300 \mathrm{mg}$ was done in group I, with garlic oil capsules (250 mg) $\mathrm{n}$ group II and nutrition counseling with special emphasis on the use of raw garlic and fish in daily diet was imparted in group III. At the end of study significant decrease was seen in mean daily intake of meat and poultry, fats and oils, sugar and jaggery, GLVs, nuts and oilseeds in all the three groups. Intake of refined cereals, roots and tubers decreased in group I and II whereas milk and milk products in group III. Significant increase was seen in intake of pulses and legumes, other vegetables, fruits in all the three groups. Significant decrease was also seen in daily intake of energy, total fat, SFA, PUFA : SFA, cholesterol, n- 6 fatty acids and n-6/n-3 ratio whereas intake of n-3 fatty acids, dietary fibre, MUFA and protein to increased in all the three groups. Significant increase in intake of cardioprotective foods was more in nutrition counseling group as subjects were advised to make dietary modifications also.
\end{abstract}

Key Words : Fish oil, Garlic oil, Nutrition Counseling, Coronary heart disease

How to cite this article : Kaur, Navjot (2014). Effect of fish oil, garlic oil supplementation and nutrition counselling on daily food and nutrient intake of at risk coronary heart subjects. Food Sci. Res. J., 5(2): 86-94. 